\title{
Efficacy of Proximal Aspiration Thrombectomy for Using Balloon-Tipped Guide Catheter in Acute Intracranial Internal Carotid Artery Occlusion
}

\author{
Yong-Won Kim, M.D., ${ }^{1,2}$ Dong-Hun Kang, M.D., ${ }^{1,3}$ Yang-Ha Hwang, M.D., Ph.D., ${ }^{2}$ Jaechan Park, M.D., Ph.D., ${ }^{3}$ Yong-Sun Kim, M.D., Ph.D. \\ Departments of Radiology, ${ }^{1}$ Neurology, ${ }^{2}$ Neurosurgery, ${ }^{3}$ Cerebrovascular Center, Kyungpook National University Hospital, School of Medicine, \\ Kyungpook National University, Daegu, Korea
}

Objective : Mechanical thrombectomy (MT) for acute intracranial internal carotid artery (ICA) occlusion is often complicated by difficult revascularization and non-involved territory embolization possibly related with larger clot-burden. This study aims to evaluate the efficacy of proximal aspiration thrombectomy (PAT) using a balloon-tipped guide catheter for clot-burden reduction in such cases with period-to-period analysis (period 1 : standard MT without PAT; period 2 : PAT first, then standard MT for the remaining occlusion).

Methods : Eighty-six patients who underwent MT for acute intracranial ICA occlusion were included in this analysis from the prospectively maintained stroke registry (33 patients in period 1 and 53 in period 2). In period 2, 'responder' was defined as a case where some amount of clot was retrieved by PAT and the following angiography showed partial or full recanalization.

Results : Fifteen of fifty-three patients in period 2 (28.3\%) were 'responders' to PAT. There was a significantly higher incidence of atrial fibrillation in the 'responder' subgroup. Period 2 showed a significantly shorter puncture-to-reperfusion time (94.5 minutes vs. 56.0 minutes; $p=0.002$ ), a significantly higher Thrombolysis in Cerebral Infarction of $2 \mathrm{~b}-3$ reperfusion (45.5\% vs. $73.6 \%$; $p=0.009$ ), but only a trend for better 3 -month favorable outcome (mRS 0-2; $36.4 \%$ vs. 54.7\%; $p=0.097$ ). There was no increase in the incidence of procedure-related complications or intracranial hemorrhage in period 2.

Conclusion : A strategy of PAT before standard MT may result in shorter puncture-to-reperfusion time and better angiographic outcome than a strategy of standard MT for acute intracranial ICA occlusion.

Key Words : Acute ischemic stroke · Balloon-tipped guide catheter · Internal carotid artery · Mechanical thrombectomy · Proximal aspiration thrombectomy.

\section{INTRODUCTION}

Although this generation of mechanical thrombectomy (MT) techniques, including stent retriever and direct thrombus aspiration, has resulted in higher recanalization rates compared to previous generations ${ }^{6,8,11,16,18,21)}$, acute internal carotid artery (ICA) occlusion with extensive clot-burden still can result in poor outcomes, perhaps as a result of difficult recanalization, higher complication rate, or non-involved-territory embolization ${ }^{4,10,19)}$. There have been a few attempts to introduce a method for reducing clot-burden by manual clot aspiration, either through a balloon-tipped guide catheter (BGC) or a non-BGC ${ }^{3,12,22)}$.

We routinely practiced proximal aspiration thrombectomy
(PAT) for clot-burden reduction in the cases of intracranial ICA occlusion since the BGC was introduced in Korea. Our method PAT involved manual clot aspiration using a $50 \mathrm{cc}$ syringe at the cervical segment ICA through a 6 Fr coaxial guide catheter, while the balloon of the outer 9 Fr BGC was inflated. After a few attempts of PAT, our standard MT involving forced arterial suction thrombectomy (FAST) with the Penumbra reperfusion catheter (Penumbra Inc., Alameda, CA, USA) and/or Solitaire stent (Covidien, Irvine, CA, USA) thrombectomy was followed to recanalize the remaining occlusion.

The primary purpose of this study was to evaluate the efficacy of the aforementioned PAT for clot-burden reduction in cases of acute intracranial ICA occlusion by comparing procedure time

• Received : November 30, 2015 • Revised : February 25, 2016 • Accepted : March 20, 2016

- Address for reprints : Dong-Hun Kang, M.D.

Departments of Radiology and Neurosurgery, Cerebrovascular Center, Kyungpook National University Hospital, School of Medicine, Kyungpook National University, 130

Dongdeok-ro, Jung-gu, Daegu 41944, Korea

Tel : +82-53-420-6246, Fax : +82-53-423-0504, E-mail : kdhdock@hotmail.com

- This is an Open Access article distributed under the terms of the Creative Commons Attribution Non-Commercial License (http://creativecommons.org/licenses/by-nc/3.0) which permits unrestricted non-commercial use, distribution, and reproduction in any medium, provided the original work is properly cited. 
and angiographic (Thrombolysis in Cerebral Infarction, TICI) $)^{5)}$ and functional (modified Rankin Scale, mRS) outcome between two treatment periods (period 1 : standard MT without PAT; period 2 : PAT first, then standard MT for the remaining occlusion).

\section{MATERIALS AND METHODS}

\section{Patient selection}

The subjects were retrospectively analyzed from a prospectively-maintained stroke registry. We reviewed 112 consecutive patients from a five-year period who received MT for acute intracranial ICA occlusion. The inclusion criteria for MT were as follows : 1) acute ischemic stroke corresponding to intracranial ICA occlusion on CT angiography or MR angiography; 2) infarction volume on DWI or CT less than half of the corresponding vascular territory. We excluded 26 patients with ICA occlusion thought to be caused by preexisting severe stenosis at the carotid bulb where urgent carotid angioplasty and/or stenting for recanalization were required. Local institutional review board approved this study for retrospective analysis.

\section{Endovascular recanalization strategy and assessment}

After inspection of treatable ICA occlusion by diagnostic angiography, MT was performed by 2 experienced neurointerventionists under local or general anesthesia. In period 1 (from May 2009 to June 2011), our standard MT with FAST using the Penumbra reperfusion catheter and/or the Solitaire stent was directly performed at the occlusion level of intracranial ICA. Techniques for FAST and Solitaire thrombectomy are the same as previously described ${ }^{8,14,18)}$. In period 2 (from July 2011 to June 2014), the MT procedure was initiated by PAT, which was comprised of manual clot aspiration using a $50 \mathrm{cc}$ syringe at the cervical segment ICA through a 100-cm long 6 Fr coaxial guide catheter (Envoy; Cordis, Miami Lakes, FL, USA) while the balloon of an outer 85-cm long 9 Fr BGC (Optimo; Tokai Medical,

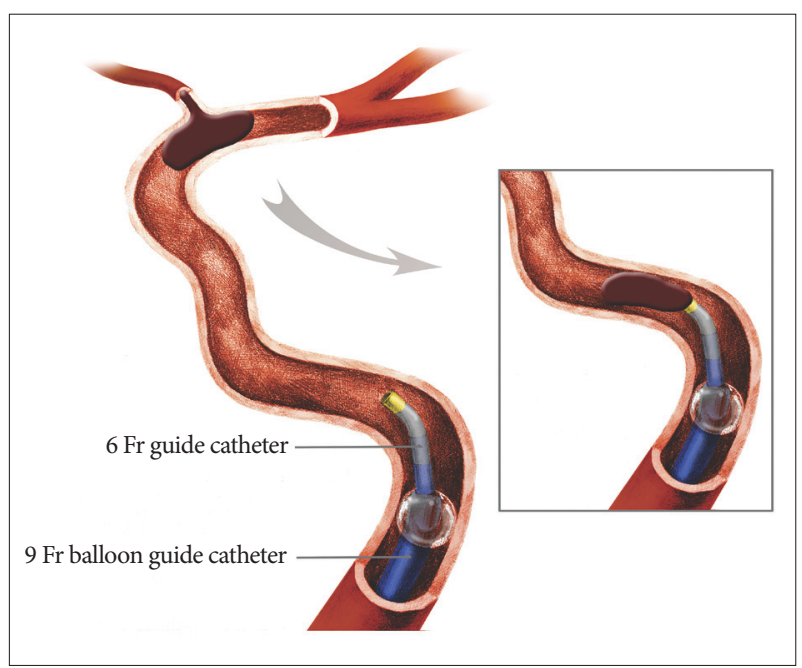

Fig. 1. Illustration of the proximal aspiration thrombectomy in an intracranial internal carotid artery occlusion.
Aichi, Japan) was inflated (Fig. 1). We defined 'responder' as a case where some amount of clot was retrieved and the following angiography showed partial or full reperfusion after PAT (Fig. 2, upper two rows). If the following angiography still demonstrated ICA occlusion after PAT, the case was regarded as 'non-responder' (Fig. 2, lower two rows). After a few attempts of PAT, subsequent MT was undertaken to recanalize the remaining occlusion using FAST and/or stent retriever. Once the Solitaire stent became available in October 2010, Solitaire stent thrombectomy followed FAST in refractory cases ${ }^{9)}$.

Neurological deficit was assessed with the National Institute of Health Stroke Scale (NIHSS) at baseline, 1 day, and 7 days. A brain CT scan was performed 24 hours after endovascular treatment and in case of neurological deterioration. Reperfusion was measured using the TICI scale and was counted as successful if TICI 2b-3 was achieved in the final angiography. Symptomatic intracranial hemorrhage (ICH) was defined as any hemorrhage with an increase in NIHSS score of 4 or more within 24 hours $^{18)}$. The mRS was used to evaluate functional outcome at 3 months after index stroke, and mRS 0-2 was defined as a favorable functional outcome.

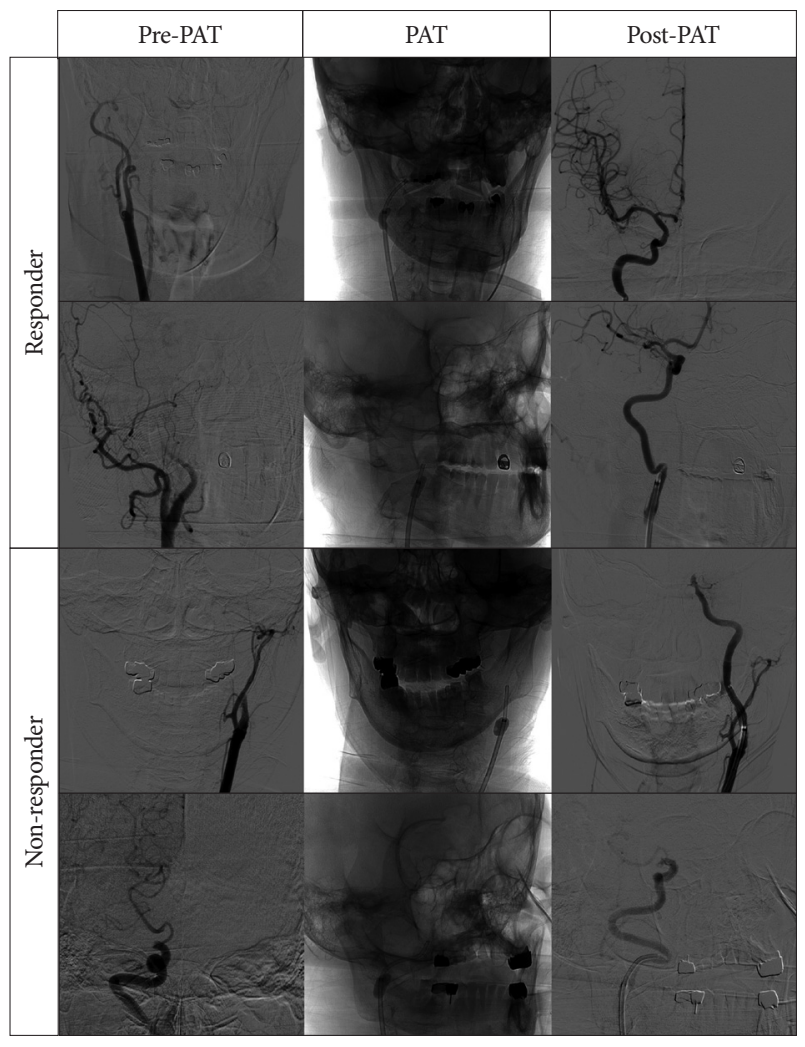

Fig. 2. This shows four typical case examples of proximal aspiration thrombectomy (PAT) for clot-burden reduction using balloon-tipped guiding catheter (BGC) in acute intracranial internal carotid artery (ICA) occlusion. The cases in the first and second row are 'responders' to the technique, which indicates that the following angiographies are showing partial or full recanalization after retrieval of some clots during the procedure. And the cases in the third and fourth row are 'non-responders' to the technique, which indicates that the following angiographies are showing still occluded ICA after a few attempts of the PAT procedure. 


\section{Statistics}

The statistical analysis was performed using the SPSS statistical package (SPSS for Windows, version 20.0, Chicago, IL, USA). The $\chi^{2}$, fisher exact, independent $t$, and Mann-Whitney $\mathrm{U}$ tests were used for comparison as appropriate.

\section{RESULTS}

Eighty-six patients with acute intracranial ICA occlusion were treated with MT and were included in this analysis. There were 33 patients in period 1 and 53 in period 2. PAT was effective in $28.3 \%(15 / 53)$ of patients in period 2, resulting in complete recanalization for 5 and incomplete recanalization for the other 10 (Fig. 2).

No clinically relevant differences were found in the baseline characteristics between the 2 groups, with the exception of door-to-puncture time (Table 1). The median puncture-to-reperfusion time was 94.5 minutes in period 1 and 56.0 minutes in period 2, a difference that was statistically significant $(p=0.002)$. TICI of $2 b-3$ reperfusion was achieved in 15 of 33 patients in period 1 and 39 of 53 patients in period 2, with significantly better reperfusion in period $2(45.5 \%$ vs. $73.6 \%$; $p=0.009)$. And also, distal embolization was non-significantly less in the period 2 (33.3\% vs. $22.6 \%$; $p=0.276$ ). There was only a trend for better 3 -month favorable outcome in period 2 (36.4\% vs. $54.7 \%$; $p=$
0.097). There were no significant differences between the 2 groups regarding the incidence of procedure-related complications and symptomatic ICH ( $p=0.556$ and $p=1.000$, respectively). In the subgroup analysis between 'responder' $(n=15)$ and 'non-responder' $(n=38)$ in period 2 (Table 2), there was a significantly higher incidence of atrial fibrillation in the responder subgroup $(86.7 \%$ vs. $57.9 \%$; $p=0.046)$. The median puncture-to-reperfusion time was significantly shorter in the responder subgroup (31 minutes vs. 71 minutes; $p=0.001$ ), and the rate of TICI $2 \mathrm{~b}-3$ reperfusion also showed a better tendency in the responder subgroup (93.3\% vs. $65.8 \% ; p=0.080)$. However, there was no significant difference in 3-month favorable outcome ( $66.7 \%$ vs. $50.0 \%$; $p=0.272$ ). Regarding the subsequent MT method for the remaining occlusion after PAT, only $6.7 \%(1 / 15)$ of responders were treated with FAST-to-Solitaire switching, compared to $52.6 \%$ (20/38) of the non-responders.

\section{DISCUSSION}

It is widely known that an occlusion at ICA location itself can be regarded as an important indicator of poor prognosis ${ }^{19}$. The presence of a larger clot-burden in the ICA occlusion compared to other locations could be one major cause of such a poor clinical course ${ }^{1)}$. A larger thrombus volume results in a smaller surface area-to-thrombus ratio at the occlusion site, which presum-

Table 1. Baseline characteristics, angiographic, and clinical outcomes in both periods

\begin{tabular}{|c|c|c|c|}
\hline Variables & Period $1(n=33)$ & Period $2(\mathrm{n}=53)$ & $p$ value \\
\hline Age, y (mean) & $67.79 \pm 11.37$ & $68.66 \pm 11.59$ & 0.733 \\
\hline Sex (female, \%) & $16(48.5)$ & $33(62.3)$ & 0.209 \\
\hline Baseline NIHSS (median) & $16(14-20)$ & $19(15-21)$ & $0.099^{*}$ \\
\hline Atrial fibrillation, $\mathrm{n}(\%)$ & $18(54.5)$ & $35(66.0)$ & 0.287 \\
\hline ICA occlusion level, n (\%) & & & 0.295 \\
\hline Cervical & $10(30.3)$ & $13(24.5)$ & \\
\hline Petro-cavernous & $13(39.4)$ & $15(28.3)$ & \\
\hline Supraclinoid-terminal & $10(30.3)$ & $25(47.2)$ & \\
\hline Intravenous tPA, n (\%) & $19(57.6)$ & $22(41.5)$ & 0.147 \\
\hline Onset to door, min (median) & $201.5(100.25-249.0)$ & $126(40-222)$ & $0.097^{\star}$ \\
\hline Door to puncture, min (median) & $113.5(89-138.25)$ & $87(70.5-101)$ & $0.001^{\star}$ \\
\hline Puncture to reperfusion, min (median) & $94.5(61.75-135.5)$ & $56(35-88)$ & $0.002^{*}$ \\
\hline TICI 2-3, n (\%) & $27(81.8)$ & $50(94.3)$ & 0.081 \\
\hline TICI $2 \mathrm{~b}-3, \mathrm{n}(\%)$ & $15(45.5)$ & $39(73.6)$ & 0.009 \\
\hline Any ICH, n (\%) & $9(28.1)$ & $19(35.8)$ & 0.463 \\
\hline Symptomatic ICH, n (\%) & $2(6.2)$ & $4(7.5)$ & 1.000 \\
\hline Favorable outcome at 3 months, $\mathrm{n}(\%)$ & $12(36.4)$ & $29(54.7)$ & 0.097 \\
\hline Mortality, n (\%) & $3(9.1)$ & $4(7.5)$ & 1.000 \\
\hline MT technique, $\mathrm{n}(\%)$ & & & 0.076 \\
\hline FAST only & $26(78.8)$ & $32(60.4)$ & \\
\hline Switching & $7(21.2)$ & $21(39.6)$ & \\
\hline Procedure related complication, $\mathrm{n}(\%)$ & $2(6.1)$ & $1(1.9)$ & 0.556 \\
\hline Distal embolization, n (\%) & $11(33.3)$ & $12(22.6)$ & 0.276 \\
\hline
\end{tabular}

${ }^{*}$ Mann-Whitney U Test. FAST : forced arterial suction thrombectomy, ICA : internal carotid artery, ICH : intracranial hemorrhage, MT : mechanical thrombectomy, NIHSS : National Institute of Health Stroke Scale, TICl : Thrombolysis in Cerebral Infarction, tPA : tissue Plasminogen Activator 
Table 2. Subgroup analysis of angiographic data between responder and non-responder in period 2

\begin{tabular}{|c|c|c|c|}
\hline Variables & Responder $(\mathrm{n}=15)$ & Non-responder $(\mathrm{n}=38)$ & $p$ value \\
\hline Intravenous tPA, n (\%) & $5(33.3)$ & $17(44.7)$ & 0.448 \\
\hline Atrial fibrillation, n (\%) & $13(86.7)$ & $22(57.9)$ & 0.046 \\
\hline ICA occlusion level, $\mathrm{n}(\%)$ & & & 0.493 \\
\hline Cervical & $3(20.0)$ & $10(26.3)$ & \\
\hline Petro-cavernous & $6(40.0)$ & $9(23.7)$ & \\
\hline Supraclinoid-terminal & $6(40.0)$ & $19(50.0)$ & \\
\hline Onset to door, min (median) & $171.5(69-231)$ & $109(39.5-215.5)$ & $0.137^{\star}$ \\
\hline Door to puncture, min (median) & $94(64-109)$ & $86(70.75-99.5)$ & $0.412^{*}$ \\
\hline Puncture to reperfusion, min (median) & $31(26-51)$ & $71(49.25-89.25)$ & $<0.001^{*}$ \\
\hline TICI $2-3, \mathrm{n}(\%)$ & $15(100)$ & $35(92.1)$ & 0.550 \\
\hline TICI 2b-3, n (\%) & $14(93.3)$ & $25(65.8)$ & 0.080 \\
\hline Favorable outcome at 3 months, $\mathrm{n}(\%)$ & $10(66.7)$ & $19(50.0)$ & 0.272 \\
\hline Symptomatic ICH, n (\%) & $0(0.0)$ & $4(10.5)$ & 0.568 \\
\hline MT technique, $\mathrm{n}(\%)$ & & & 0.002 \\
\hline FAST only & $14(93.3)$ & $18(47.4)$ & \\
\hline Switching & $1(6.7)$ & $20(52.6)$ & \\
\hline
\end{tabular}

${ }^{*}$ Mann-Whitney U Test. FAST : forced arterial suction thrombectomy, ICA : internal carotid artery, ICH : intracranial hemorrhage, MT : mechanical thrombectomy, TICI : Thrombolysis in Cerebral Infarction

ably can decrease the efficacy of intravenous thrombolysis with tissue-type plasminogen activator and $\mathrm{MT}^{2,13,20)}$. In addition, the potential for non-target embolization of uninvolved territories can be another practical concern during MT in such cases ${ }^{10)}$. To date, timely recanalization is the only procedure that has been shown to improve outcome ${ }^{7,17}$. In that regard, there have been several attempts to reduce clot-burden during MTs for acute ICA occlusions to achieve better angiographic and functional outcome $^{3,12,22)}$.

In our practice, standard MT using FAST and/or Solitaire stent was directly undertaken at the occlusion level of the intracranial ICA in period 1 . However in period 2 , we routinely began with PAT for clot-burden reduction in such cases, which featured manual clot aspiration at the cervical segment ICA with a 6 Fr coaxial guide catheter through a 9 Fr BGC; then we followed with the standard MT as in period 1 for the cases of remaining occlusions. This study highlights the role of PAT with BGC by comparing variables between the 2 periods with different MT protocols for intracranial ICA occlusions.

The main findings of this study were the following : 1) there was a significantly shorter puncture-to-recanalization time ( $p=0.003$ ), a significantly higher rate of TICI $2 \mathrm{~b}-3$ recanalization $(p=0.009)$, but only a trend for better 3-month favorable outcome ( $p=0.097$ ) in period 2 compared to period $1 ; 2$ ) PAT was effective in $28.3 \%$ (15/53) of the patients in period 2 , where complete recanalization occurred in 5 and incomplete recanalization in the other 10 (Fig. 2); 3) in the subgroup analysis between 'responder' and 'non-responder' in period 2, there was a higher incidence of atrial fibrillation $(p=0.046)$ and a significantly shorter puncture-to-recanalization time $(p=0.008)$ in responder subgroup; on the other hand, there was only a trend for higher TICI $2 \mathrm{~b}-3$ recanalization $(p=0.080)$ and no signifi- cant differences in 3-month favorable outcome ( $p=0.272$ ).

Regarding the MT techniques used for any remaining occlusion, there was a significantly higher number of FAST-to-Solitaire switching in non-responder subgroup $(1 / 15,6.7 \%$ in responder vs. $20 / 38,52.6 \%$ in non-responder, $p=0.002)$. This can be explained by noting that most of the subsequent MT in the responder subgroup was simply finished without additional Solitaire thrombectomy; but in the non-responder subgroup, the following MT was not always easy, and $52.6 \%$ required FAST-to-Solitaire switching. As previously published ${ }^{9)}$, FASTto-Solitaire switching can play some role in achieving better angiographic and functional outcome in difficult cases. Thus, we hypothesized that more case numbers of switching in the nonresponder subgroup could be one possible explanation of the non-significant differences in TICI $2 \mathrm{~b}-3$ recanalization and 3-month favorable outcome in the subgroup analysis between 'responder' and 'non-responder'.

PAT can have several advantages. First, this procedure can be applied easily and quickly. This just requires the advancement of a 6 Fr guide catheter through a 9 Fr BGC, followed by manual aspiration. Two or three attempts of this procedure take only a few minutes; and if the case is refractory, an institutional MT protocol can easily follow. Second, this method was safe without elevating procedure-related complications or ICH. This required no advancement of additional devices to the intracranial artery; thus, the chance of complications may be minimal. Third, this can be more effective than the historically-performed clot aspiration technique under manual compression of the common carotid artery ${ }^{15,22)}$ because a BGC can make a definite proximal flow arrest. Finally, we can apply an intermediate guide catheter; for example, Distal Access Catheter (Concentric Medical, Mountain View, CA, USA) or Navien catheter (Covi- 
dien, Mansfield, MA, USA), as a substitute for the 6 Fr guide catheter during PAT, which may be more effective because such catheters can be advanced more distally.

The application of BGC played an important role for the PAT and subsequent standard MT. First, in the general aspiration thrombectomy, aspiration using pump or syringe was performed after contact between thrombus and reperfusion catheter. However, PAT was performed distant proximal area without facing between thrombus and reperfusion catheter. Therefore, proximal flow arrest by BGC could establish temporary closed space, which was theoretically better circumstance for PAT. Second, during subsequent standard MT, the rate of distal embolization was non-significantly less in the Period 2 (33.3\% vs. 22.6, $p=0.276$ ). Although similar MT strategy was applied between periods, time from groin puncture to recanalization was shorter in the period 2. And also, in the comparison between period 1 and non-responder of period 2, time from groin puncture to recanalization revealed shorter tendency in the non-responder of period 2 (94.5 min vs. $71.0 \mathrm{~min}, p=0.052$ ) and successful recanalization rate demonstrated higher tendency ( $45.5 \%$ vs. $65.85 \%, p=0.085$ ). These results were comparable with previous studies $^{14,15)}$.

However, this study has several potential limitations. Although the patients were enrolled prospectively, the data were analyzed retrospectively. Therefore, the treatment groups were not randomized and concurrent. And, as we previously discussed, the difference in number of switching cases between both periods could be a bias in the interpretation of the present data. In addition, there was a baseline imbalance for door-topuncture time between the two periods. The door-to-puncture time was significantly reduced in period 2 , which might have resulted from increased experience at the system level. Therefore, we could not exclude the possibility that this imbalance influenced the outcome.

\section{CONCLUSION}

The present study compared two different strategies for intracranial ICA occlusion with similar baseline characteristics. Based upon the data, PAT effective in $28.3 \%$ of the cases, which resulted in shorter puncture-to-recanalization times and significantly higher TICI $2 \mathrm{~b}-3$ recanalization rates without increasing the incidence of procedure-related complications or ICH. However, these results should be interpreted cautiously because of the aforementioned limitations, and confirmation by prospective multi-center trials seems necessary.

\section{- Acknowledgements}

This research was supported by Kyungpook National University Research Fund, 2012.

\section{References}

1. Barreto AD, Albright KC, Hallevi H, Grotta JC, Noser EA, Khaja AM, et al. : Thrombus burden is associated with clinical outcome after intraarterial therapy for acute ischemic stroke. Stroke 39: 3231-3235, 2008

2. Bhatia R, Hill MD, Shobha N, Menon B, Bal S, Kochar P, et al. : Low rates of acute recanalization with intravenous recombinant tissue plasminogen activator in ischemic stroke : real-world experience and a call for action. Stroke 41 : 2254-2258, 2010

3. Eesa M, Almekhlafi MA, Mitha AP, Wong JH, Goyal M : Manual aspiration thrombectomy through balloon-tipped guide catheter for rapid clot burden reduction in endovascular therapy for ICA L/T occlusion. Neuroradiology 54 : 1261-1265, 2012

4. Gralla J, Burkhardt M, Schroth G, El-Koussy M, Reinert M, Nedeltchev $\mathrm{K}$, et al. : Occlusion length is a crucial determinant of efficiency and complication rate in thrombectomy for acute ischemic stroke. AJNR Am J Neuroradiol 29: 247-252, 2008

5. Higashida RT, Furlan AJ, Roberts H, Tomsick T, Connors B, Barr J, et al. : Trial design and reporting standards for intra-arterial cerebral thrombolysis for acute ischemic stroke. Stroke 34 : e109-e137, 2003

6. Hong KS, Ko SB, Lee JS, Yu KH, Rha JH : Endovascular recanalization therapy in acute ischemic stroke : updated meta-analysis of randomized controlled trials. J Stroke $17: 268-281,2015$

7. Hwang YH, Kang DH, Kim YW, Kim YS, Park SP, Suh CK : Outcome of forced-suction thrombectomy in acute intracranial internal carotid occlusion. J Neurointerv Surg 5 Suppl 1 : i81-i84, 2013

8. Kang DH, Hwang YH, Kim YS, Park J, Kwon O, Jung C : Direct thrombus retrieval using the reperfusion catheter of the penumbra system : forced-suction thrombectomy in acute ischemic stroke. AJNR Am J Neuroradiol 32 : 283-287, 2011

9. Kang DH, Kim YW, Hwang YH, Park J, Hwang JH, Kim YS : Switching strategy for mechanical thrombectomy of acute large vessel occlusion in the anterior circulation. Stroke 44: 3577-3579, 2013

10. King S, Khatri P, Carrozella J, Spilker J, Broderick J, Hill M, et al. : Anterior cerebral artery emboli in combined intravenous and intra-arterial rtPA treatment of acute ischemic stroke in the IMS I and II trials. AJNR Am J Neuroradiol 28 : 1890-1894, 2007

11. Lee HG, Rhim JK, Kim YH, Sheen SH, Oh SH, Chung BS : The comparison of outcome between thromboaspiration and aggressive mechanical clot disruption in treating hyperacute stroke patients. J Korean Neurosurg Soc 50 : 311-316, 2011

12. Lutsep HL, Clark WM, Nesbit GM, Kuether TA, Barnwell SL : Intraarterial suction thrombectomy in acute stroke. AJNR Am J Neuroradiol $23: 783-786,2002$

13. Machi P, Costalat V, Lobotesis K, Maldonado IL, Vendrell JF, Riquelme C, et al. : Solitaire FR thrombectomy system : immediate results in 56 consecutive acute ischemic stroke patients. J Neurointerv Surg $4: 62$ 66, 2012

14. Mokin M, Setlur Nagesh SV, Ionita CN, Levy EI, Siddiqui AH : Comparison of modern stroke thrombectomy approaches using an in vitro cerebrovascular occlusion model. AJNR Am J Neuroradiol 36 : 547 551, 2015

15. Nguyen TN, Malisch T, Castonguay AC, Gupta R, Sun CH, Martin CO, et al. : Balloon guide catheter improves revascularization and clinical outcomes with the Solitaire device : analysis of the North American Solitaire Acute Stroke Registry. Stroke 45 : 141-145, 2014

16. Nogueira RG, Lutsep HL, Gupta R, Jovin TG, Albers GW, Walker GA, et al. : Trevo versus Merci retrievers for thrombectomy revascularisation of large vessel occlusions in acute ischaemic stroke (TREVO 2) a randomised trial. Lancet $380: 1231-1240,2012$

17. Rha JH, Saver JL: The impact of recanalization on ischemic stroke outcome : a meta-analysis. Stroke 38 : 967-973, 2007

18. Saver JL, Jahan R, Levy EI, Jovin TG, Baxter B, Nogueira RG, et al. : Solitaire flow restoration device versus the Merci Retriever in patients with acute ischaemic stroke (SWIFT) : a randomised, parallel-group, non-in- 
feriority trial. Lancet $380: 1241-1249,2012$

19. Smith WS, Lev MH, English JD, Camargo EC, Chou M, Johnston SC, et al. : Significance of large vessel intracranial occlusion causing acute ischemic stroke and TIA. Stroke 40 : 3834-3840, 2009

20. Tan IY, Demchuk AM, Hopyan J, Zhang L, Gladstone D, Wong K, et al. : $\mathrm{CT}$ angiography clot burden score and collateral score : correlation with clinical and radiologic outcomes in acute middle cerebral artery infarct. AJNR Am J Neuroradiol 30 : 525-531, 2009
21. Turk AS, Spiotta A, Frei D, Mocco J, Baxter B, Fiorella D, et al. : Initial clinical experience with the ADAPT technique : a direct aspiration first pass technique for stroke thrombectomy. J Neurointerv Surg 6 : 231237, 2014

22. Xu GF, Suh DC, Choi CG, Kim JK, Kim W, Kim SJ, et al. : Aspiration thrombectomy of acute complete carotid bulb occlusion. J Vasc Interv Radiol 16 : 539-542, 2005 